\title{
DEVELOPMENT OF SELF NANO-EMULSIFYING DRUG DELIVERY SYSTEM FOR AN ANTI- HYPERTENSIVE AGENT FELODIPINE: A SYSTEMATIC APPROACH FOR LIPID NANO- FORMULATION WITH IMPROVED ORAL BIOAVAILABILITY IN RATS
}

\section{K. TRIDEVA SASTRI ${ }^{1 *}$, G. V. RADHA ${ }^{1}$}

${ }^{1}$ GITAM Institute of Pharmacy, GITAM Deemed to be University, Rushikonda, Visakhapatnam, Andhra Pradesh State, India Email: trideva.k@gmail.com

Received: 19 Feb 2020, Revised and Accepted: 27 Mar 2020

\section{ABSTRACT}

Objective: The present study involves the development of SNEDDS employing essential oils for enhancing biopharmaceutical performance.

Methods: Preliminary investigations suggested the selection of cinnamon oil as an essential oil, tween 60 as a surfactant, while transcutol HP as a cosolvent for formulating SNEDDS. Formulations evaluated for stability, robustness to dilution, and emulsification time, droplet size, zeta potential (ל), cloud point, in vitro drug release, drug excipient compatibility, TEM, stability assessment and in vivo pharmacokinetic performance in rats.

Results: All formulations were robust, stable, and revealed excellent emulsification time $<40 \mathrm{~s}$, with fine droplet size $(11.41 \pm 2.41 \mathrm{~nm})$, lower PDI (0.028-0.277). Formulation F(FLD)6 exhibited a release of $97.7 \%$ within 4 h, and TEM photograph confirmed spherical droplets. The bioavailability results revealed a higher rate and extent of absorption, AUC, and Cmax for the formulations found to be 1212.4 and $355.40 \pm 13.67$ ( $<<0.05$ ). The results recommend that the developed formulation approach offers bioavailability enhancement of FLD.

Conclusion: The study concluded that SNEDDS would be an effective formulation system in increasing the aqueous solubility and potentially bioavailability. Furthermore, it can be applied for other therapeutic categories of drugs belonging to BCS class II and IV that show comparable biopharmaceutical challenges.

Keywords: Felodipine, essential oils, Bioavailability enhancement, Self-nano emulsifying systems, Pharmacokinetic study

(C) 2020 The Authors. Published by Innovare Academic Sciences Pvt Ltd. This is an open access article under the CC BY license (http://creativecommons.org/licenses/by/4.0/) DOI: http://dx.doi.org/10.22159/ijap.2020v12i3.37203. Journal homepage: https://innovareacademics.in/journals/index.php/ijap

\section{INTRODUCTION}

Felodipine (FLD) is a Biopharmaceutical Classification System (BCS) class II, belonging to a therapeutic category of anti-hypertensive [1]. Due to its susceptibility towards hepatic metabolism and lipophilicity, FLD has poor oral bioavailability. Palpably, the oral route is preferred by its potential physiological aids, besides patient compliance. Various formulation systems were developed for enhancing FLD oral bioavailability, including microparticles, solid dispersions, and nanoparticles [2,3].

In recent times, oral lipid-based formulation systems have gained much significance in enhancing the solubility, followed by oral bioavailability of lipophilic molecules [4]. Lipid-based systems vary from simple to complex isotropic mixtures of oils, surfactants, and co-surfactant/cosolvents [5]. Apart from enhancing solubility, these systems also tend to improve absorption by several auxiliary mechanisms like inhibition of efflux transport mediated by Pglycoprotein, promoting lymphatic transport surpassing the firstpass metabolism and enhancing membrane permeability $[6,7]$.

Self-nano-emulsifying drug delivery system (SNEDDS) is one of the most effective approaches amongst lipid-based systems for enhancing solubility and the bioavailability of lipophilic molecules [8]. SNEDDS is a simple isotropic blend of oil, surfactant, and cosurfactant/cosolvent and drug, which spontaneously yield rapid ultrafine $\mathrm{O} / \mathrm{W}$ nanoemulsion under mild agitation and upon subsequent dilution in gastro-intestinal fluids with a droplet size range of $<100 \mathrm{~nm}$, aiding large surface for effective release of drug for absorption [9]. Similarly, to the enhanced solubility and dissolution, bioavailability can also be influenced by uptake by lymphatic transport.

Our current study aims to formulate FLD loaded SNEDDS for enhancement of its solubility, dissolution. Subsequently, to enhance oral bioavailability by employing essential oils as a capable excipient for self-emulsification. Comparative evaluation of optimized formulation with FLD-suspension for their in vitro drug release and in vivo pharmacokinetic study in rats was studied.

\section{MATERIALS AND METHODS}

\section{Materials}

FLD was a generous gift from Aurobindo Pharma Ltd., (Hyderabad, India). Caproyl $90^{\circledR}$, Transcutol $\mathrm{HP}^{\circledR}$, Labrafil M $1944{ }^{\circledR}$, Labrasol $^{\circledR}$ were kindly gifted by Gattefosse India Pvt Ltd (Mumbai, India). Clove oil, Polyethylene glycol-200 Molychem (Mumbai, India), Anise Oil, Cinnamon Oil, Orange Oil, Lemon Oil Genuine Chemicals Co. (Mumbai, India), Peppermint Oil Yuuca Enterprises (Mumbai, India), Tween-60 Sisco Research Laboratory (Mumbai, India). Dialysis membrane (MWCO 12 to $14 \mathrm{KD}$ ) procured from Himedia (Mumbai, India). All other reagents and chemicals were stringently used of analytical grade and used as received.

\section{Methods}

Saturation solubility study

The saturation solubility of FLD has been carried out in several oils, surfactants and co-surfactant/co-solvents using a water bath shaker $[10,11]$. FLD was added in an excess amount to each of closed glass vials containing about $2 \mathrm{ml}$ of each vehicle. The attained mixtures were initially vortexed for $5 \mathrm{~min}$ to facilitate solubilization. The blends were placed in a controlled temperature of $37 \pm 0.5^{\circ} \mathrm{C}$ in a water bath shaker (REMI, India) for a period of 72 $\mathrm{h}$ to obtain equilibrium. Then, the samples were subjected to centrifugation at $4500 \mathrm{rpm}$ for $20 \mathrm{~min}$ to separate the undissolved drug. The supernatant was filtered through $0.45 \mu \mathrm{m}$ (Whatman, USA) membrane filter. All filtrates were suitably diluted with methanol and analyzed spectrophotometrically at $\lambda_{\max } 238 \mathrm{~nm}$ by UV-vis spectrophotometer (Shimadzu, Japan).

\section{Pseudo-ternary phase diagram study}

From the results of the saturation solubility, miscibility, and emulsification efficacy, cinnamon oil as oil phase, tween 60 as surfactant and transcutol HP as co-solvent selected for SNEDDS formulation. The phase diagrams of oil, surfactant-co-solvent mixture $\left(\mathrm{S}_{\mathrm{mix}}\right)$ and double distilled water plotted using a water 
titration method. The ratio of surfactant to co-surfactant varied from $1: 3$ to $3: 1$. For each pseudo ternary phase diagram at a specific surfactant/cosurfactant weight ratio, the blends of oil, surfactant and cosurfactant prepared with the ratio of oil to the bends of surfactant and cosurfactant at 9:1 to $1: 9[12,13]$. All the ratios studied for visual clarity and spontaneity of emulsification. The blends considered to be good when a clear and transparent emulsion was formed spontaneously without any phase separation on standing [14]. In order to optimize the concentration selection of the nanoemulsion region form the phase diagrams was since solution remain clear regardless of infinite dilution. The phase diagrams plotted using ProSim ternary diagram software to detect nano emulsifying region.

\section{Preparation of FLD loaded SNEDDS}

FLD is most frequently available in the dose of $10 \mathrm{mg}$, FLD loaded SNEDDS prepared in the doses of $10 \mathrm{mg}$. The preparation of SNEDDS achieved with simple blending where the FLD was dissolved in an isotropic mixture of lipid, surfactant and cosolvent with vortex shaker for $5 \mathrm{~min}$, to acquire a homogeneous mixture. A total of ten formulations with $10 \mathrm{mg}$ of FLD prepared to scrutinize through spontaneous emulsification method, which was further characterized for kinetic stability, visual observation, robustness to dilution, emulsification, droplet size, zeta potential (ל), cloud point, in vitro drug release, drug excipient compatibility, TEM, stability assessment and in vivo pharmacokinetic performance in rats. The composition of the FLD loaded SNEDDS is exhibited in (table 1).

Table 1: Formulation composition of FLD loaded SNEDDS (\%V/V)

\begin{tabular}{llll}
\hline Formulation code & Components (\% V/V) & Tween 60 & Transcutol HP \\
\cline { 2 - 4 } & Cinnamon Oil & 69.4 & 23.1 \\
F(FLD)1 & 7.5 & 67.5 & 22.5 \\
F(FLD)2 & 10 & 65.5 & 22 \\
F(FLD)3 & 12.5 & 63.75 & 21.25 \\
F(FLD)4 & 15 & 61.9 & 20.6 \\
F(FLD)5 & 17.5 & 60 & 20 \\
F(FLD)6 & 20 & 58.14 & 19.36 \\
F(FLD)7 & 22.5 & 56.25 & 18.75 \\
F(FLD)8 & 25 & 54.38 & 18.12 \\
F(FLD)9 & 27.5 & 52.5 & 17.5 \\
F(FLD)10 & 30 & \\
\hline
\end{tabular}

\section{Kinetic stability studies}

Nanoemulsions are said to be kinetically stable systems. The chosen ten formulations were imperilled to kinetic stability by employing heating and cooling cycle and centrifugation tests. Formulations which were found kinetically stable were selected for further evaluation [15].

\section{Heating-cooling cycle}

The cycles involved six cycles were the formulations were kept at refrigerator temperature, 5 and 45 for $48 \mathrm{~h}$ for each cycle. The formulations scrutinized for drug precipitation and phase separation. Further, all formulations diluted with distilled water (1:50), and resulting nanoemulsions examined for instability glitches - those formulations which are stable at these temperatures further subjected to centrifugation test.

\section{Centrifugation test}

In this assessment, all formulations were diluted with distilled water (1:50) and subjected to centrifugation at $3500 \mathrm{rpm}$ for $30 \mathrm{~min}$. Post centrifugation, the formulations were screened for instability like phase separation, creaming or cake formation. Such unstable formulations excluded from further studies, and stable formulations carried out for further parameters.

\section{Robustness to dilution}

This study involved the assessment of formulations for physical changes upon diluting by 50,100 -and 1000 -fold with distilled water, $0.1 \mathrm{~N} \mathrm{HCl}$ and phosphate buffer (pH 6.8), respectively. Post dilution, the visual aspect was judged after $2 \mathrm{~h}$. Furthermore, the resultant nanoemulsions were kept for $24 \mathrm{~h}$ and observed for any physical changes like phase separation, cloudiness or precipitation. All the formulations which yield clear nanoemulsions at different dilution pleats in examined media were said to pass this test [16].

\section{Self-emulsification efficiency}

The rate of emulsification (self-emulsification time) has been defined as the efficiency of the formulations to disperse upon aqueous dilution conferring to the method described by [17]. About $1 \mathrm{ml}$ of each formulation was added dropwise to $250 \mathrm{ml}$ of phosphate buffer (pH 6.8) in USP dissolution apparatus II (Electrolab TDT 06L). The paddle speed was adjusted to $50 \mathrm{rpm}$ to facilitate mild agitation with the temperature-maintained $37 \pm 5$ 圆 the self-emulsification time given by the time required for the preconcentrate to yield homogeneous dispersion. Formulations passing the test carried out for further studies.

\section{Characterization of the SNEDDS formulations}

Based on the results of prior studies, six of the formulations (F(FLD)1, F(FLD)2, F(FLD)3, F(FLD)4, F(FLD)5 and F(FLD)6) were selected for further characterization.

\section{Cloud point measurement}

Each formulation was diluted with distilled water at a ratio of 1:50 and placed in a water bath with a slow rise in temperature (5 0/min). Cloud point was measured as the temperature at which the first sign of turbidity was visually observed [18].

Determination of zeta potential (Ъ); mean droplet size and polydispersity index

Zeta potential means droplet size and polydispersity index of nanoemulsions were determined post-100-fold dilution of selected formulations with distilled water were determined by dynamic light scattering (DLS) technique using Malvern Zetasizer Nano (Malvern Instruments, UK).

\section{In vitro drug release study}

This study involved the use of the dialysis bag method (Dialysis membrane MWCO 12 to $14 \mathrm{KD}$, Himedia., India) with some modifications $[19,20]$. The drug release was estimated by diluting from each formulation (equivalent to $5 \mathrm{mg}$ FLD) with phosphate buffer ( $\mathrm{pH}$ 6.8), then was loaded into the pre-soaked membrane and clamped on both sides. The release medium $(500 \mathrm{ml}$ of phosphate buffer $\mathrm{pH}$ 6.8) was added to the apparatus. The secured membrane was tied to the paddle of the apparatus USP dissolution apparatus II (Electrolab TDT 06L) and allowed to rotate freely. The experiment was thermostatically controlled at $37 \pm 0.5^{\circ} \mathrm{C}$, and stirred $50 \mathrm{rpm}$. At predetermined time intervals, $5 \mathrm{ml}$ of sample was withdrawn from the medium and immediately replenished with the same volume of fresh medium. All the samples were analysed spectrophotometrically with necessary dilutions at $\lambda_{\max } 238 \mathrm{~nm}$. The release patterns compared to that of pure drug suspension (with an equivalent drug concentration of test formulations). 


\section{Thermal analysis}

The endothermic melting temperature for pure FLD powder, FLD loaded SNEDDS was determined using DSC (DSC-TA instruments USA Q10). The pure FLD and FLD loaded SNEDDS were respectively packed in an aluminium pan and the thermogram was recorded with a rate of $10{ }^{\circ} \mathrm{C} / \mathrm{min}$, over a temperature range of $50-300{ }^{\circ} \mathrm{C}$ under a nitrogen atmosphere using the empty pan as the reference.

\section{Fourier-transform infrared (FTIR) spectroscopy}

The probable interactions between the drug and employed excipients were studied by FTIR spectroscopy. The IR spectra of pure FLD powder and FLD loaded SNEDDS were carried out using Bruker Vertex 70 FT-IR spectrometer (Bruker, USA). The samples were prepared by $\mathrm{KBr}$ pellet method. The spectrum was recorded from regions of $4000 \mathrm{~cm}^{-1}$ to $400 \mathrm{~cm}^{-1}$ [21].

\section{Transmission electron microscopy (TEM)}

In this investigation, the morphology of FLD loaded SNEDDS studied using transmission electron microscopy after a 1000 -fold dilution with distilled water using JEM 2100 (JOEL, Japan). On a copper grid, one drop of the sample was deposited and allowed to stand for 5 min. The excess was removed with a blotting paper. The prepared grid was latter negatively stained with a drop of phosphotungstic acid $(1 \% \mathrm{w} / \mathrm{v})$ for $30 \mathrm{~s}$. The grid was air-dried and then was observed by TEM.

\section{In vivo study}

The pharmacokinetic study was carried out in male Wistar rats weighing 200-250 g. The Institutional Animal Ethical Committee of GITAM Institute of Pharmacy, India, approved the study (Reg. no. IAEC/GIP-1287/KTS-S/Approved/9/2019). All the rats were clinically healthy during the entire study and strictly housed under standard conditions of temperature and humidity. The in vivo bioavailability studies for both FLD-suspension and F (FLD)6 carried out in two groups $(\mathrm{n}=6)$. Food was withdrawn $12 \mathrm{~h}$ prior to the study water was provided ad libitum and no food was allowed post-dosing until the end of the study (after $8 \mathrm{~h}$ ). Each of the animals received a single dose $(1 \mathrm{mg} / \mathrm{kg})$ of FLD-suspension as a pure drug with a group I and the SNEDDS formulation F (FLD) 6 with group II orally with the help of oral feeding needle. Blood samples $(0.5 \mathrm{ml})$ were collected at predetermined intervals of predose $0 \mathrm{hr}$ and $0.5,1$, $2,3,4,6$, and $8 \mathrm{hr}$ post oral dose administration in pre-coated K2EDTA tubes. The samples were centrifuged at $4500 \mathrm{rpm}$ for 20 min, and the separated plasma samples stored at refrigerated conditions (2-4 [? ) until analysis. FLD concentrations in plasma samples were determined suitably by HPLC method [22].

\section{RESULTS AND DISCUSSION}

\section{Saturation solubility study}

The pertinent solubility of a drug substance in the excipients is categorically crucial for developing a successful formulation besides avoiding drug precipitation during in situ self-emulsification in the gut region. The (fig. 1) exemplifies the saturation solubility data of FLD in various essential oils, surfactants and co-solvents. Among various essential oils employed, the highest solubility was observed in cinnamon oil (i.e. $281 \pm 1.07 \mathrm{mg} / \mathrm{ml}$ ). Similarly, among surfactants and co-solvents employed tween $60 \quad(152 \pm 1.22 \mathrm{mg} / \mathrm{ml})$ and transcutol HP (i.e. $363 \pm 1.29 \mathrm{mg} / \mathrm{ml}$ ) exhibited the highest solubilization capacity and spontaneity of the emulsification ability. As a result of low toxicity and higher stability to the effect of $\mathrm{pH}$ and ionic strength when compared to ionic and amphiphilic surfactants, nonionic surfactants are typically preferred [20]. In addition, the higher solubility of the drug in nonionic surfactants can be described as a result of higher HLB value, which enables faster solubilization of lipophilic drugs and imparts spontaneous emulsification ability [23]. Transcutol HP was selected as a co-solvent, which furthermore reduces the interfacial tension and improves the solubility of FLD.

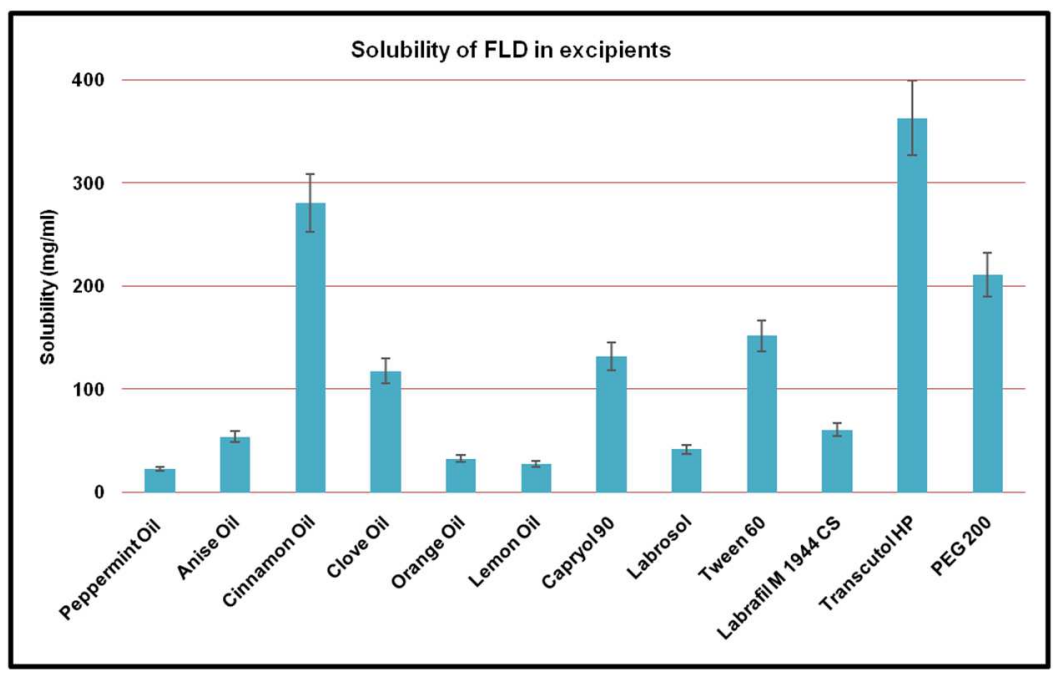

Fig. 1: Saturation solubility of FLD in different vehicles (mean $\pm S D(N=3)$ )

\section{Pseudo ternary phase diagram study}

To identify the self-emulsifying regions, pseudo-ternary plots were constructed. Clear isotropic regions determine the self-emulsifying regions which were recognized by optical observation. Besides, it also helps in defining the appropriate excipient ratios for the development of formulations that guarantee the spontaneity of in situ self-emulsification in the gut region [24].

Fig. 2(A-E) demonstrates the pseudo-ternary diagrams constructed for demarcating the suitable nanoemulsion region for FLD loaded SNEDDS. The shaded region specifies the self-emulsifying region, and the higher area indicated for cinnamon oil and $S_{\text {mix }}$ ration of $3: 1$ containing tween 60-transcutol HP. As it is understood, that all surfactants significantly irritate and are poorly tolerated, hence large magnitudes of surfactants may lead to irritation in the GI tract, and therefore ideally emulsifying systems with more substantial portions of essential oils are selected [25]. In the case of selfemulsifying systems, free energy required is very minimal, as surfactants play a vital role in minimizing the interfacial tension to form kinetically stable emulsions [26]. The studies were also carried to explore the effects of $S_{\text {mix }}$ ratios 1:3, 1:2, 1:1, and 2:1, after observing the clarity, stability for $48 \mathrm{~h}$, revealed that the nanoemulsion region further increases in 3:1 ratio, owing to increase in the surfactant concentration in $S_{\text {mix }}$ ratio has increased the nanoemulsion region, and this can be explained owing to higher HLB value (i.e. 14.9) of tween 60 which readily enhances the emulsification efficiency for forming nanoemulsions. 
In brief, amid various $S_{\text {mix }}$ ratios explored, the ratio $3: 1$, was selected for the formulation of the self-emulsifying system. Ten formulations were selected for the drug incorporation and further study. These mixtures were identified within the nanoemulsion region of studied pseudo-ternary plots, and the compositions of the ten formulations loaded with FLD is shown in (table 1).

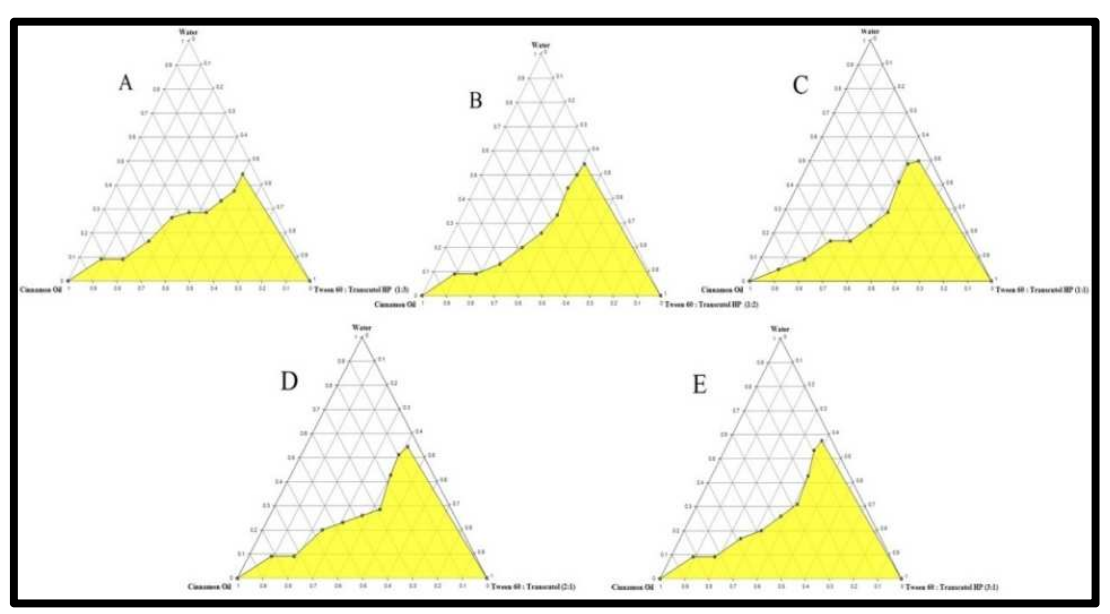

Fig. 2: Phase behaviour of nanoemulsion formed from cinnamon oil with Tween 60: Transcutol HP and water varying Tween 80: Transcutol HP ratio as (A) 1:3; (B) 1:2; (C) 1:1; (D) 2:1; (E) 3:1

\section{Kinetic stability}

As the SNEDDS forms nanoemulsion spontaneously, the formulation should possess the ability to withstand stability against creaming, precipitation and caking. The FLD loaded systems portrayed excellent stability. All dispersions were clear and retained their visual facet during the study signifying their nanoemulsion nature. Accordingly, the study has not revealed any distress to the applied conditions and confirmed the stability of the prepared formulations.

\section{Robustness to dilution}

Robustness to dilution helps to understand the drug precipitation at higher dilutions, as such precipitations alter and reduce the in vivo fate of drug absorption. All the prepared formulations were exposed to different folds of dilutions in various media (distilled water, $\mathrm{pH} 6.8$ and $\mathrm{pH} 1.2$ ) to mimic the in vivo conditions and to ensure the formation of a uniform formulation. Almost all formulations exhibited to be robust to the dilutions except four formulations (F(FLD)7 to F(FLD)10 showed immediate milky dispersions. The higher concentrations of oil components could lead to loss of clarity [27]. Formulations (F(FLD)1 to F(FLD)6, were determined to retain their clarity at different dilution volumes of various media thus confirming their robustness.

\section{Self-emulsification efficiency}

Post-oral administration the formulations are exposed to the in-situ gut conditions where the formulations experience aqueous dilution, thereby the preparations should immediately disperse under mild agitation provided by the peristaltic agitations to yield a fine nanoemulsion. Therefore, self-emulsification time is a significant assessment parameter for self-emulsification efficiency. Amid the developed formulations, six formulations (F(FLD)1 to F(FLD)6 were determined to be of grade A regardless of the dispersion media.

Irrespective of the dispersing medium, all formulations portrayed a short emulsification time of $<40$ (s) (table 2), confirming their high emulsification efficiency. Formulations with higher concentrations of essential oil required a longer time to get completely dispersed. Subsequently, formulations exhibited increased spontaneity for nanoemulsion formation as the concentration of $S_{\text {mix }}$ increases in the formulations. One reason could be extreme penetration of aqueous phase into the oil phase triggering substantial interfacial disruption, and thereby droplets moved into the bulk aqueous phase.

Table 2: Self-emulsification time for selected formulations in different dispersing media

\begin{tabular}{|c|c|c|c|}
\hline \multirow[t]{2}{*}{ Formulation code } & \multicolumn{3}{|l|}{ Self-emulsification time (s) ${ }^{*}$} \\
\hline & Phosphate buffer (pH 6.8) & Distilled water & $0.1 \mathrm{~N} \mathrm{HCl}(\mathrm{pH} \mathrm{1.2)}$ \\
\hline $\mathrm{F}(\mathrm{FLD}) 1$ & 8 & 9 & 11 \\
\hline $\mathrm{F}(\mathrm{FLD}) 2$ & 12 & 11 & 13 \\
\hline $\mathrm{F}(\mathrm{FLD}) 3$ & 16 & 15 & 18 \\
\hline $\mathrm{F}(\mathrm{FLD}) 4$ & 19 & 21 & 22 \\
\hline $\mathrm{F}(\mathrm{FLD}) 5$ & 24 & 25 & 28 \\
\hline F(FLD)6 & 33 & 29 & 35 \\
\hline
\end{tabular}

*Data expressed as mean of 3

\section{Characterization of the SNEDDS formulations}

Along with robustness to dilution those formulations that exhibited good kinetic stability and showed high emulsification efficiency with excellent visual grade post dilutions, namely (F(FLD)1 to F(FLD)6 carried for further characterization.

\section{Cloud point measurement}

The temperature above which the dispersion turns turbid is said to be cloud point, and this is due to dehydration of polyethylene oxide chains of the non-ionic surfactants leading to lowered HLB values.
For the selected formulations, cloud points were (table 3) very high; hence were determined to be competent for body temperature, therefore, circumventing phase separation in the GIT. Higher cloud point temperature could be attributed to the solubility of the drug in the formulation components, with an optimized ratio of $S_{\text {mix }}$ along with higher HLB values.

Determination of zeta potential (Ъ); mean droplet size and polydispersity index

The droplet size and the interfacial area are inversely proportional; therefore, the smaller droplet size results in better diffusion and 
absorption owing to the increased interfacial area. Accordingly, the droplet size distribution of nanoemulsions post-self-emulsification becomes significant is assessing the in vivo performance of the formulation [28].

The mean droplet size of the formulations (table 3) from the study confirmed that the formulations showed droplet size in the nanometric range (fig. 3). All formulations exhibited minor PDI values indicating excellent uniformity, $\mathrm{S}_{\text {mix }}$ may act synergistically to lower the interfacial tension, consequently yield fine nanoemulsion.

As a result of the ultrafine emulsion system, which imparts a robustness to flocculation or towards coalescence, making the system kinetically stable. The kinetic stability becomes significant in long term physical stability.

Table 3: Cloud point, droplet size, zeta potential, and PDI for selected formulations

\begin{tabular}{llll}
\hline Formulation code & Cloud point $\left({ }^{\circ} \mathrm{C}\right)$ & Droplet size (nm) & Zeta potential (mV) \\
\hline F(FLD)1 & 89 & $9.680 \pm 1.72$ & $-3.65 \pm 1.97$ \\
F(FLD)2 & 86 & $9.917 \pm 2.12$ & $-3.68 \pm 1.49$ \\
F(FLD)3 & 82 & $10.17 \pm 2.62$ & $-5.90 \pm 1.66$ \\
F(FLD)4 & 80 & $10.39 \pm 1.69$ & $-4.84 \pm 2.32$ \\
F(FLD)5 & 75 & $11.10 \pm 1.33$ & $-6.87 \pm 1.52$ \\
F(FLD)6 & 73 & $11.41 \pm 2.41$ & $-7.22 \pm 2.81$ \\
\hline
\end{tabular}

${ }^{*}$ Data expressed as $($ mean $\pm \mathrm{SD}(\mathrm{N}=3))$

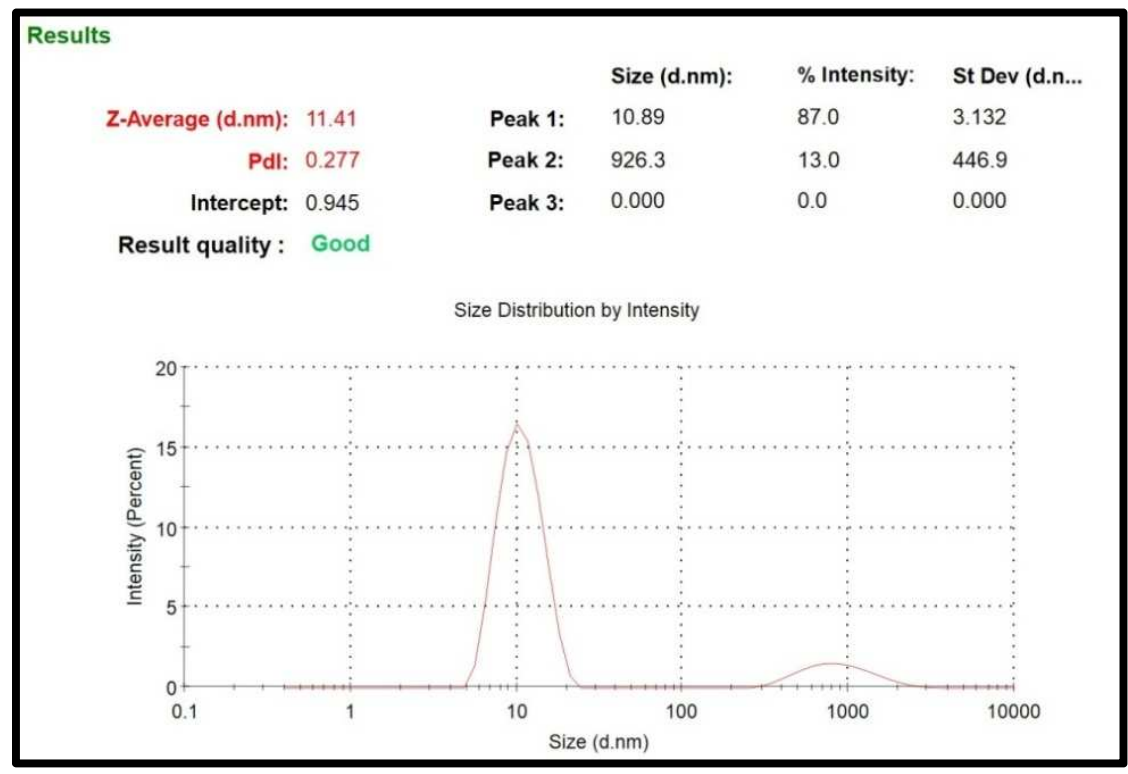

Fig. 3: Droplet size distribution of FLD-loaded nanoemulsion F(FLD)6

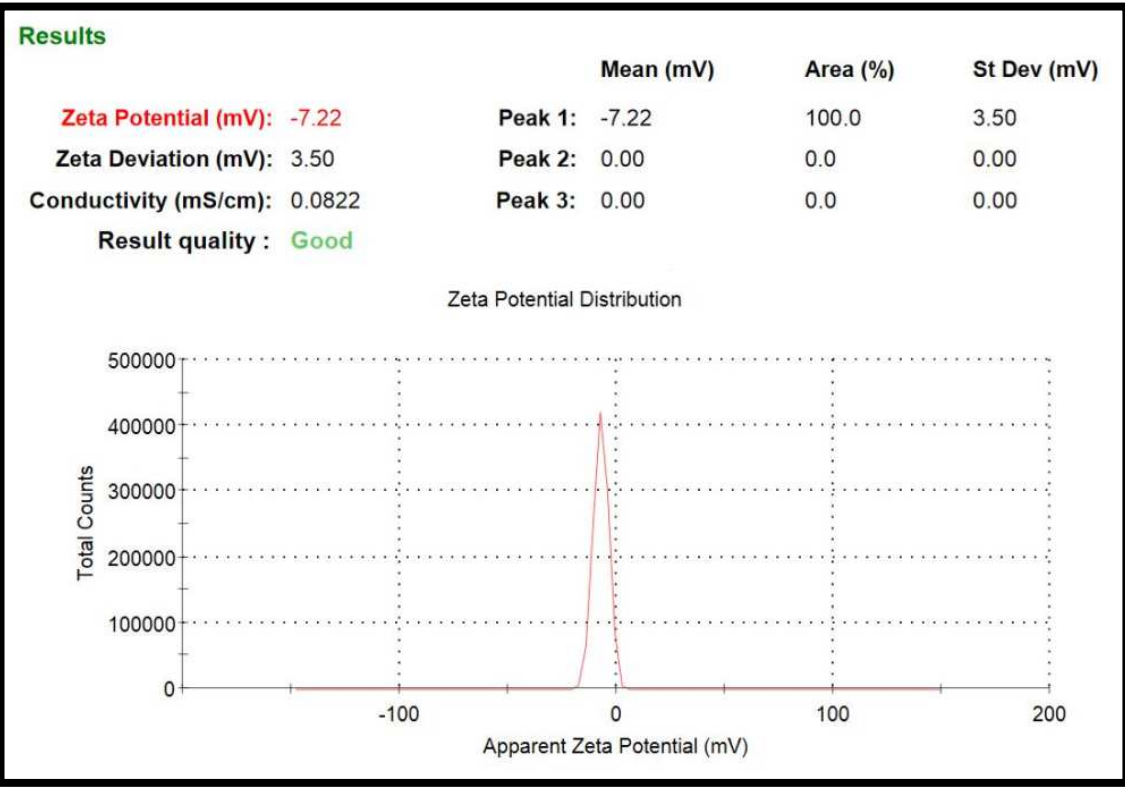

Fig. 4: Zeta potential (ל) mV of FLD-loaded nano-emulsion F(FLD)6 
The mean droplet size for formulations varied insignificantly compared to all formulations F(FLD)5 and F(FLD)6, exhibited slightly larger mean droplet size when compared to other formulations. Majorly due to higher essential oil concentration compared to other formulations.

All the selected formulations exhibited negative zeta potential values (table 3) (fig. 4). The zeta potential is interrelated to the electrostatic repulsions and aggregation of the droplets. Coalescence is inhibited with the higher values of zeta potential, thus enabling the stability of emulsions [29]. The negative charges attributed to ionization free fatty acid chains present in the compositions of the formulation system.

\section{In vitro release study}

Only when the free drug molecules get released from the entrapped droplets of nanoemulsion system, evaluation can be achieved. Thus, the formulation system cannot be assessed by the conventional release approach. Therefore, the dialysis bag method adapted for the study [30]. The comparative study of release profiles exhibited definite improvement in the drug release rate from the formulations when compared to the pure drug suspension. Release patterns of the formulations along with FLD suspension, are shown in (fig. 5). The higher release from the formulation system may be due to the higher availability of dissolved FLD from the droplets that provides a larger surface area for its release.

Drug release from F(FLD)6 was significantly higher compared to other formulations owing to entrapment of free drug in the lipid, followed by spontaneous emulsification enabled by emulsifying agents. An almost complete release of $97.7 \%$ was achieved by the best formulation F(FLD) 6 within $4 \mathrm{~h}$, whereas only $12 \%$ of drug release was achieved from a drug suspension. The results significantly endorse marked improvement of FLD solubilization with full potential for enhancing the oral bioavailability.

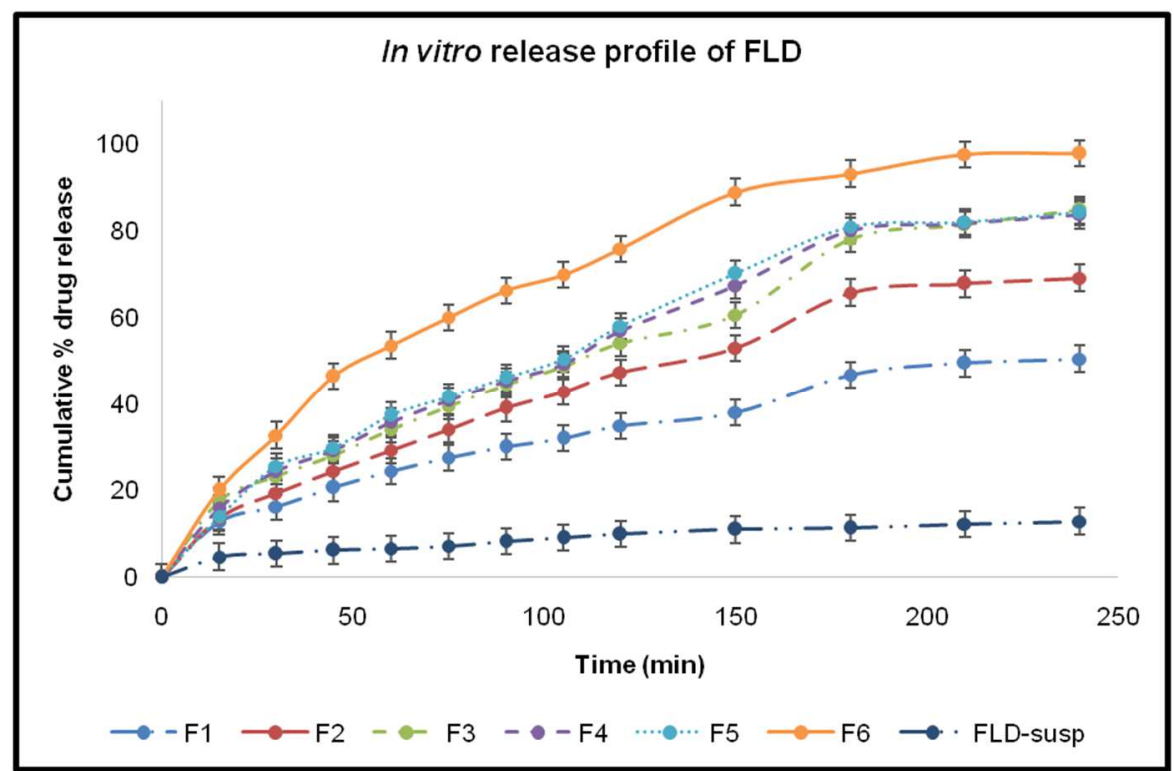

Fig. 5: In vitro (\%) cumulative drug release profile of FLD loaded SNEDDS and FLD-suspension in phosphate buffer 6.8 (mean \pm SD (N=3))

\section{Thermal analysis}

The DSC thermograms of pure drug sample and the FLD loaded-SNEDDS formulations shown in (fig. 6a and 6b). Pure drug powder exhibited a sharp endothermic peak at $145.36{ }^{\circ} \mathrm{C}$, with almost concurrent to the melting point confirming its purity, suggesting the crystallinity of drugs. Concerning to the formulation mixture, exhibited broad endothermic peak and drifted little ahead by approximately $15^{\circ} \mathrm{C}$, maybe after FLD blended with the lipid excipients, it is likely that FLD was in an amorphous, molecularly dispersed state in the lipid of the formulation.

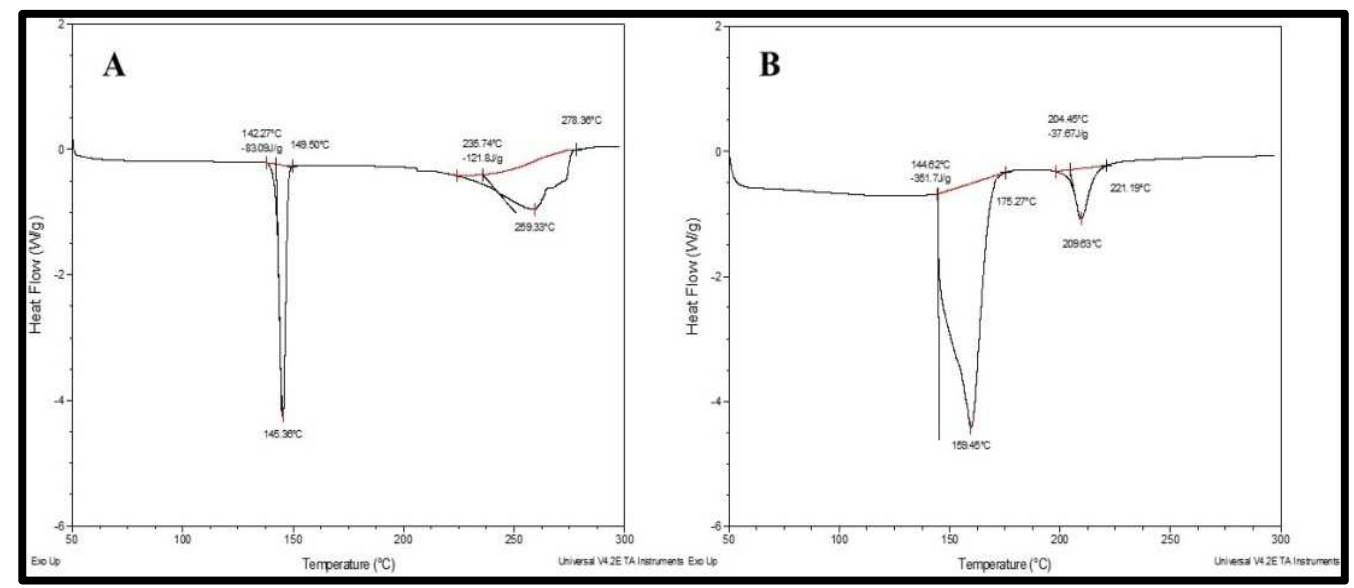

Fig. 6: (a) DSC thermogram of FLD pure drug, (b) DSC thermogram of FLD loaded nano-formulation mixture 


\section{Fourier-transform infrared (FTIR) spectroscopy}

The FTIR spectra for FLD and its SNEDDS are shown in (fig. 7a and 7b). FTIR spectra of FLD exhibited characteristic peaks of C-H Strat $3523.69 \mathrm{~cm}^{-1}$, Aromatic-Str at $3071.21 \mathrm{~cm}^{-1}, \mathrm{C}=0$ (carboxylate) at
$1620.20 \mathrm{~cm}^{-1}$, C-N Strat $1099.42 \mathrm{~cm}^{-1}$, Aromatic C-H bending at 616.82 $\mathrm{cm}^{-1}$. Correspondingly the FTIR spectra of SNEDDS also showed all these characteristic peaks with minor shifts. These results from FTIR spectral analysis confirmed that there was no chemical interaction between drug and excipients used in the formulation.

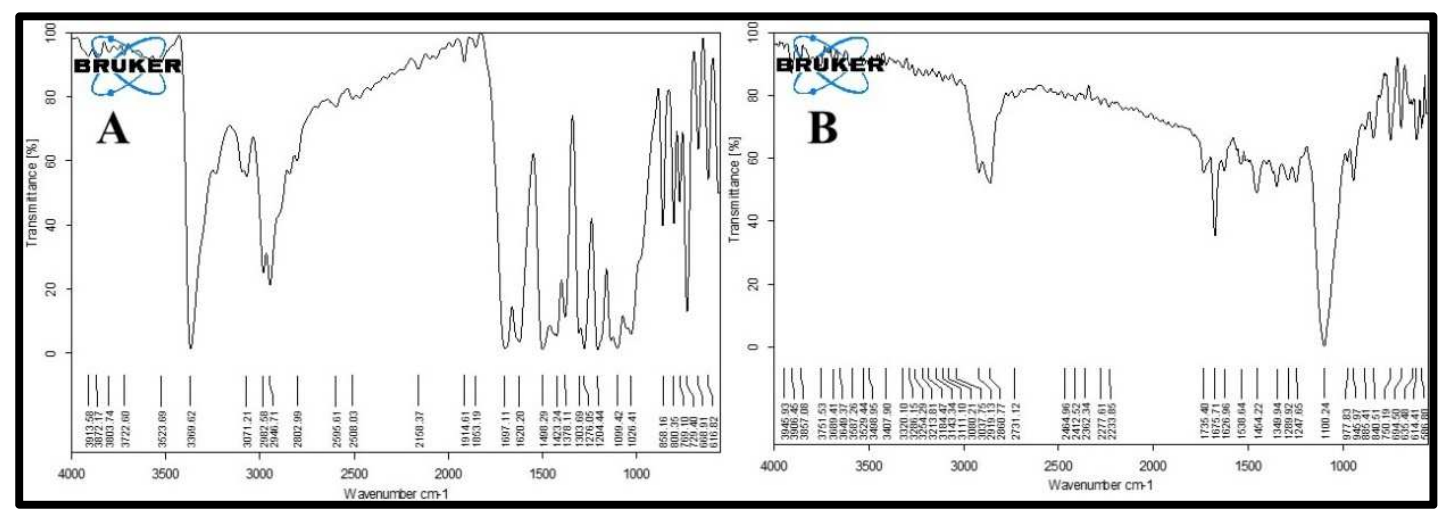

Fig. 7: (a) FTIR spectra of FLD pure drug, (b) FTIR spectra of FLD loaded nano-formulation mixture

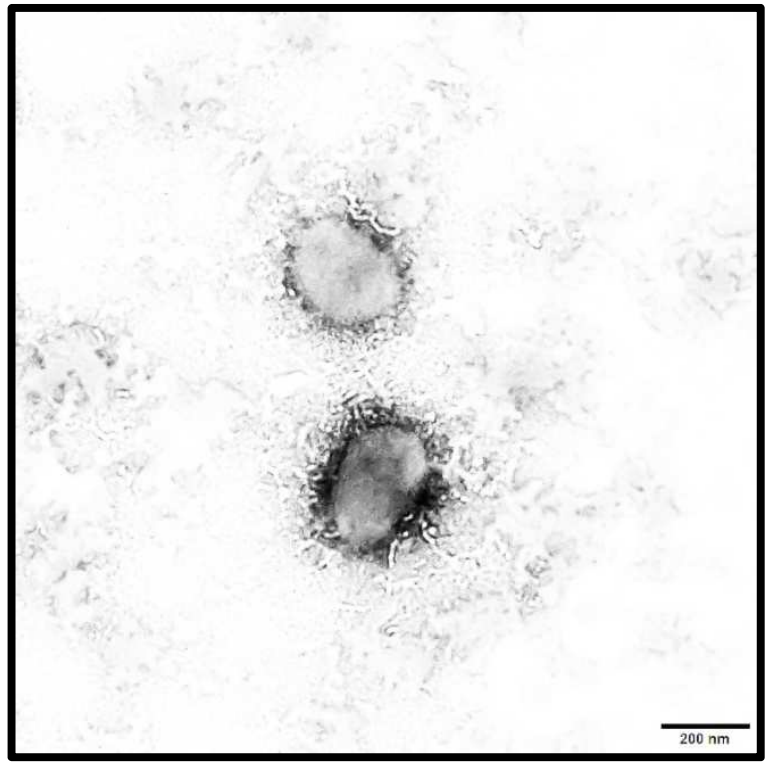

Fig. 8: TEM photograph of (F(FLD)6) nano-formulation

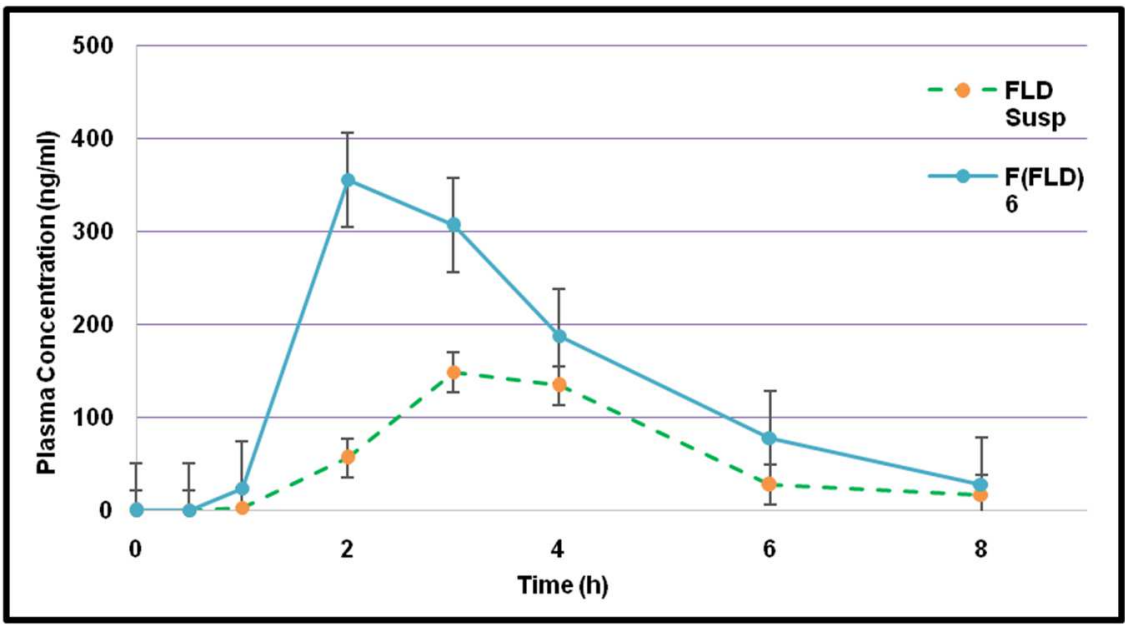

Fig. 9: Plasma concentrations of FLD following oral administration of (A) FLD Susp and (B) F(FLD)6 (mean \pm SD (N=6)) 


\section{Transmission electron microscopy (TEM)}

The surface morphology of the reconstituted formulation observed using TEM (fig. 8). The TEM photograph confirms that all droplets were homogenous, spherically shaped with good integrity and these nanosized droplets had no sign of aggregation, besides photograph did not reveal any precipitation of FLD.

\section{In vivo study}

The in vivo pharmacokinetic behaviour of the FLD loaded SNEDDS was assessed and compared with the pure drug suspension following oral administration to the two groups of Wistar rats $(n=6)$. The plasma concentration versus time profiles of FLD in rats for formulations system and drug suspension represented in (fig. 9). The results showed that the $[\mathrm{AUC}]_{0}^{\infty}$ and $\mathrm{C}_{\max }$ for the SNEDDS formulation found to be 1212.4 and $355.40 \pm 13.67$ when compared to the drug suspension, which was found to be 518.7 and $148.93 \pm 8.92$, respectively. The per cent relative bioavailability of SNEDDS, when compared with the FLD suspension, was found to be 245.68 , which suggests the significant improvement of the rate and extent of absorption. Besides, the Tmax was slightly longer for drug suspension when compared to the formulation system. Significant differences found between the two formulations regarding $[\mathrm{AUC}]_{0}^{\infty}$ and $\mathrm{C}_{\max }$ parameters at $\mathrm{p}<0.05$.

\section{CONCLUSION}

The present study effectively endorsed successful development and formulation of self-nanoemulsifying system as one of the promising delivery systems for bioavailability enhancement of FLD. Following evaluations, the formulation composed of cinnamon oil with $20 \% \mathrm{v} / \mathrm{v}$, tween 60 with $60 \% \mathrm{v} / \mathrm{v}$ and transcutol HP with $20 \% \mathrm{v} / \mathrm{v}$ exhibited best results and no incompatibles observed. Essential oils were determined to be one of the most proficient oil phases for selfemulsifying systems and may have significant bioactive effects. The formulation enhanced the in vitro release approximately eight times. It provided an effective release when compared to a drug suspension.

Furthermore, in vivo studies revealed that best formulation exhibited a higher degree of absorption when compared to drug suspension with a relative bioavailability of $245.68 \%$. The study concluded that SNEDDS would be an effective formulation system in increasing the aqueous solubility and potentially bioavailability. Furthermore, the formulation strategy may be applied for other therapeutic categories of drugs belonging to BCS class II and IV that show comparable biopharmaceutical challenges.

\section{ACKNOWLEDGMENT}

The authors would like to acknowledge and thank Gattefosse India Pvt Ltd., for their gift samples of Caproyl 90, Transcutol HP, Labrafil M 1944 CS, and Labrasol, respectively. The authors are grateful to GITAM Institute of Pharmacy, GITAM (Deemed to be University), Visakhapatnam for providing necessary facilities and support.

\section{AUTHORS CONTRIBUTIONS}

All the authors have contributed equally.

\section{CONFLICT OF INTERESTS}

The authors report no conflict of interest. This research did not receive any specific grant from any funding agencies.

\section{REFERENCES}

1. Saltiel E, Ellrodt AG, Monk JP, Langley MS. Felodipine. A review of its pharmacodynamic and pharmacokinetic properties, and therapeutic use in hypertension. Drugs 1988;36:387-428.

2. Bazzo GC, Caetano DB, Boch ML, Mosca M, Branco LC, Zétola M, et al. Enhancement of felodipine dissolution rate through its incorporation into $\partial$ E-PHB polymeric microparticles: in vitro characterization and investigation of absorption in rats. J Pharm Sci 2012;101:1518-23.

3. Sarode AL, Malekar SA, Cote C, Worthen DR. Hydroxypropyl cellulose stabilizes amorphous solid dispersions of the poorly water-soluble drug felodipine. Carbohydr Polym 2014;112:512-9.
4. Pouton CW. Formulation of poorly water-soluble drugs for oral administration: physicochemical and physiological issues and the lipid formulation classification system. Eur J Pharm Sci 2006;29:278-87.

5. Parmar N, Singla N, Amin S, Kohli K. Study of cosurfactant effect on nano emulsifying area and development of lercanidipine loaded (SNEDDS) self nano emulsifying drug delivery system. Colloids Surf B 2011;86:327-38.

6. Humberstone AJ, Charman WN. Lipid-based vehicles for the oral delivery of poorly water-soluble drugs. Adv Drug Delivery Rev 1997;25:103-28.

7. Holm R, Porter CJ, Edwards GA, Müllertz A, Kristensen HG, Charman WN. Examination of oral absorption and lymphatic transport of halofantrine in a triple-cannulated canine model after administration in self-microemulsifying drug delivery systems (SMEDDS) containing structured triglycerides. Eur J Pharm Sci 2003;20:91-7.

8. Kang BK, Lee JS, Chon SK, Jeong SY, Yuk SH, Khang G, et al. Development of self-microemulsifying drug delivery systems (SMEDDS) for oral bioavailability enhancement of simvastatin in beagle dogs. Int J Pharm 2004;274:65-73.

9. Bandopadhyay S, Singh B, Kapil R, Singh R, Katare OP. Selfemulsifying drug delivery systems (SEDDS): formulation development, characterization, and applications. Crit Rev Ther Drug Carrier Syst 2009;26:427-521.

10. Balakrishnan P, Lee BJ, Oh DH, Kim JO, Lee YI, Kim DD, et al. Enhanced oral bioavailability of coenzyme Q10 by selfemulsifying drug delivery systems. Int J Pharm 2009;74:66-72.

11. Madhavi $\mathrm{K}$, Shikha A, Suresh B. Formulation and in vitro characterization solid self-emulsifying drug delivery system of ramipril prepared by adsorption technique. Int J Curr Pharm Sci 2016;8:40-5.

12. Constantinides PP, Welzel G, Ellens H, Smith PL, Sturgis SS, Yiv $\mathrm{SH}$, et al. Water-in-oil microemulsions are containing mediumchain fatty acids/salts: formulation and intestinal absorption evaluation. Pharm Res 1996;13:210-5.

13. Srikanth reddy S, Suresh G. Design and evaluation of self-nano emulsifying drug delivery systems of manidipine for enhancement of solubility. Asian J Pharm Clin Res 2019;12:288-95.

14. Zhang P, Liu Y, Feng N, Xu J. Preparation and evaluation of selfmicroemulsifying drug delivery system of oridonin. Int J Pharm 2008;355:269-76.

15. Negi LM, Tariq M, Talegaonkar S. Nanoscale self-emulsifying oil-based carrier system for improved oral bioavailability of camptothecin derivative by P-glycoprotein modulation. Colloids Surf B 2013;111:346-53.

16. Elnaggar YS, El-Massik MA, Abdallah OY. Self-nano emulsifying drug delivery systems of tamoxifen citrate: design and optimization. Int J Pharm 2009;380:133-41.

17. Khoo SM, Humberstone AJ, Porter CJH, Edwards GA, Charman WN. Formulation design and bioavailability assessment of lipidic self-emulsifying formulations of halofantrine. Int J Pharm 1998;167:155-64.

18. Madhavi K, Shikha A, Yadav JK. Self-nano emulsifying drug delivery system of ramipril: formulation and in vitro evaluation. Int J Pharm Pharm Sci 2016;8:291-6.

19. Agrawal AG, Kumar A, Gide PS. Self-emulsifying drug delivery system for enhanced solubility and dissolution of glipizide. Colloids Surf B: Biointerfaces 2015;126:553-60.

20. Wu W, Wang Y, Que L. Enhanced bioavailability of silymarin by self-microemulsifying drug delivery system. Eur J Pharm Biopharm 2006;63:288-94.

21. Hai Xia Z, Jie XW, Zhi Bing Z, Yuan L, Zhi Gang S, Jian Feng C. Micronization of atorvastatin calcium by antisolvent precipitation process. Int J Pharm 2009;374:106-13.

22. Sahu BP, Das MK. Preparation and in vitro/in vivo evaluation of felodipine nanosuspension. Eur J Drug Metab Pharmacokinet 2013;39:183-93.

23. McConville C, Friend D. Development and characterization of a self-micro emulsifying drug delivery systems (SMEDDSs) for the vaginal administration of the antiretroviral UC-781. Eur J Pharm Biopharm 2013;83:322-9. 
24. Palamakula A, Khan MA. Evaluation of cytotoxicity of oils used in coenzyme Q10 self-emulsifying drug delivery systems (SEDDS). Int J Pharm 2004;273:63-73.

25. Pouton CW. Formulation of self-emulsifying drug delivery systems. Adv Drug Delivery Rev 1997;25:47-58.

26. Anton N, Gayet P, Benoit JP, Saulnier P. Nano-emulsions and nanocapsules by the PIT method: an investigation on the role of the temperature cycling on the emulsion phase inversion. Int J Pharm 2007;344:44-52.

27. Davies JT. Drop sizes of emulsions related to turbulent energy dissipation rates. Chem Eng Sci 1985;40:839-42.
28. Anton N, Gayet P, Benoit JP, Saulnier P. Nano-emulsions and nanocapsules by the PIT method: an investigation on the role of the temperature cycling on the emulsion phase inversion. Int Pharm 2007;344:44-52.

29. Craig DQM, Barker SA, Banning D, Booth SW. An investigation into the mechanisms of self-emulsification using particle size analysis and low-frequency dielectric spectroscopy. Int J Pharm 1995;114:103-10.

30. Griesser J, Hetenyi G, Kadas H, Demarne F, Jannin V, Bernkop Schnürch A. Self-emulsifying peptide drug delivery systems: how to make them highly mucus permeating. Int J Pharm 2018;538:159-66. 